\title{
Corrigendum
}

\section{Behavior of expanded polystyrene (EPS) blocks under cyclic pavement foundation loading}

S. M. A. Ghotbi Siabil, S. N. Moghaddas Tafreshi, A. R. Dawson and M. Parvizi Omran

REFERENCE: Ghotbi Siabil, S. M. A., Moghaddas Tafreshi, S. N., Dawson, A. R. and Parvizi Omran, M. (2019). Behavior of expanded polystyrene (EPS) blocks under cyclic pavement foundation loading. Geosynthetics International, 26, No. 1, 1-25. [https://doi.org/10.1680/jgein.18.00033]

The following changes are made to the paper.

$$
\sigma_{c}=8.02 \rho-79.31
$$

Table 2. Physical and mechanical properties of EPS geofoam

\begin{tabular}{|l|l|l|l|}
\hline \multirow{2}{*}{ Engineering properties } & \multicolumn{3}{|c|}{ Values for } \\
\cline { 2 - 4 } & EPS 20 & EPS 30 & EPS 40 \\
\hline Real density $\left(\mathrm{kg} / \mathrm{m}^{3}\right)$ & $17 \sim 19$ & $27 \sim 29$ & $37 \sim 39$ \\
Angle of internal friction $\left(^{\circ}\right)$ & $\sim 2$ & $\sim 3$ & $\sim 3.5$ \\
Apparent cohesion $(\mathrm{kPa})$ & $\sim 40$ & $\sim 70$ & $\sim 95$ \\
Elastic modulus $(\mathrm{MPa})$ & 0.81 & 2.16 & 2.86 \\
Compressive strength $(\mathrm{kPa})$ & 83.67 & 156.4 & 244.14 \\
\hline
\end{tabular}

The authors apologise for these errors. 